\title{
Ocupaciones de montaña en el marco de los primeros grupos agro-pastorales del Pirineo Central en el VI-V milenio cal ANE: una perspectiva paleoeconómica
}

\author{
Mountain occupations of the first agro-pastoral communities of the Central \\ Pyrenees during the 6-5 $5^{\text {th }}$ millennia cal BC: a paleoeconomic perspective
}

PALABRAS CLAVES: Pirineos, Neolítico, paleoecónomia, caza, agricultura.

GAKO-HITZAK: Pirinioak, Neolitikoa, paleoekonomia, ehiza, nekazaritza.

KEY WORDS: Pyrenees, Neolithic, Palaeoconomy, hunting farming.

\section{Niccoló MAZZUCCO (1), Ignacio CLEMENTE CONTE(2) Ermengol GASSIOT BALBE் ${ }^{(3)}$ y David RODRÍGUEZ ANTÓN(2,3)}

\section{RESUMEN}

En este trabajo vamos a realizar una revisión de las evidencias disponibles sobre las primeras ocupaciones de las zonas de media y alta montaña del Pirinceo Central por parte de grupos agro-pastorales. En particular, nos vamos a fijar sobre dos practicas economicas relevantes: la caza y el cutlivo de cereales. Nuestro objectivo es entender el rol que estas dos actividades tuvieron dentro del sistema económico de los primeros pobladores neolíticos y como se integraron dentro del sistema. Nuestros resultados sugieren que la caza fue solo marginalmente practicada, mientras que para la agricultura únicamente tenemos evidencias del consumo de cereales, pero por ahora no de su cultivo en zonas de alta y media montaña. Este escenario, nos acerca a comunidades agro-pastorales móviles, que se desplazarón sobre un territorio relivamente amplio para desarollar sus practicas economicas y explotar diferentes recursos.

\begin{abstract}
LABURPENA
Lan honetan, nekazaritako eta artzaintzako taldeek Erdialdeko Pirinioetako erdi mailako mendietan eta goi-mendietan egindako lehen okupazioei buruz eskuragarri dauden ebidentzien inguruko berrikusketa egingo dugu. Zehatzago esanda, bi jarduera ekonomiko esanguratsutan oinarrituko gara: ehiza eta zerealen laborantza. Gure helburua bi jarduera horiek Neolitikoko lehen biztanleen sistema ekonomikoaren baitan izan zuten rola eta sistema baitan nola txertatu ziren ulertzea da. Gure emaitzen arabera, ehiza oso gutxi egin zuten; aldiz, nekazaritzarekin lotuta, zerealen kontsumoaren inguruko ebidentziak bakarrik ditugu, baina oraingoz ez dugu erdi mailako mendiko eta goi-mendiko eremuetan halakoak egin izanaren inguruko zantzurik. Testuinguru hori ikusita, nekazaritza eta artzaintzako komunitate mugigarriak izan zirela esan dezakegu. Nahiko zabala zen lurraldean mugitzen ziren beren jarduera ekonomikoak garatzeko eta baliabide ugariak ustiatzeko.
\end{abstract}

\begin{abstract}
In this paper, we present a review of the available information about the first agro-pastoral communities which occupied the Central Pyrenees, between the $6^{\text {th }}$ and the $5^{\text {th }}$ millennium cal BC. Our aim is to discuss some aspects related to the paleoeconomical behaviours of such first settlers, with particular attention to two relevant productive activities: hunting and crop-husbandry. Both are frequently claimed to be used in mountainous areas in integration to domestic animal herding. However, to which extent both economic processes were carried out at midand high-altitudes? Do were they intensively or marginally practiced? Which was their role within the overall economic system? In this review, we will take in consideration all the available information in the Pyrenean sites; however, most of them have been only partially or fragmentarily published.

Our results suggest that both activities were only marginally practiced in the mountainous areas of the Pyrenees. Despite both hunting and crop-husbandry can be considered everyday activities, commonly represented within the archaeological records of the period, they do not appear to be intensively carried in at higher altitudes. Hunting appears only occasionally practiced as a secondary source of food gathering. Neolithic groups occupying the mid-and high-altitude of the Pyrenees do not seem to rely on hunting more than the communities settled at lower altitude; on the contrary the consumption of wild games appears, generally, lower. For what concern crop-husbandry, on the basis of the available data, we must conclude that there are no clear evidences that cereal cultivation has been carried out at mountain altitude, at least during Early Neolithic. Despite that, the archaeological record testifies the consumption of agricultural provisions -carried into the site from elsewhere and not produced or processed locally- in several cave-sites located at mountain and subalpine altitudes. This pattern suggests that, in the mountainous areas of the Central Pyrenees, crops were mainly exploited as integrative alimentary sources.
\end{abstract}

\footnotetext{
(1) UMR 7055 Préhistoire et Technologie, Maison Archéologie Ethnologie. 21 Allée de l'Université. 92023 Nanterre cedex. Correo electrónico: niccolo. mazzucco@mae.u-paris10.fr; (2) CSIC - Institución Milá y Fontanals (IMF), Departamento de Arqueología y Antropología. C/ Egipciaques, 15. 08001 Barcelona. Correo electónico: ignacio@imf.csic.es.

(2) Universitat Autònoma de Barcelona UAB. Departament de Prehistòria. Edifici B. 08193. Bellaterra (Cerdanyola del Valles), Barcelona. Correos electrónicos: ermengol.gassiot@uab.cat; david.anton79@gmail.com.

(3) Universitat Autònoma de Barcelona UAB. Departament de Prehistòria. Edifici B. 08193. Bellaterra (Cerdanyola del Valles), Barcelona. Correos electrónicos: ermengol.gassiot@uab.cat; david.anton79@gmail.com;
} 
In conclusion, our data indicate the existence of mobile herding groups which gradually occupied the mountainous areas of the Pyrenees starting from the second half of the $6^{\text {th }}$ millennium cal BC. Such groups moved over relatively large territories to develop their economic practices. Hunting and agriculture were integrated within this system and provided integrative alimentary sources and/or provisions which were moved and transported from one site to another. Nevertheless, further investigations are needed to deepen our understanding of the economic and social organization of such communities.

\section{1.- INTRODUCCIÓN}

Los primeros indicios de poblaciones agro-pastorales en el área pirenaica se fechan alrededor de 5500 cal ANE. Se trata de una serie de asentamientos aislados, localizados en las sierras pre-pirenaicas exteriores, en zonas de piedemonte o de fondo de valle a bajas y medias altitudes (200-700 msnm). La Cueva de Chaves en la Sierra de Guara en Aragón (BALDELLOU et al. 2012, MAZZUCCO et al. 2015), el poblado al aire libre de la Font del Ros (MORA et al. 1991) y la Bauma del Serrat del Pont (ALCALDE et al. 2005) en las sierras pre-pirenaicas catalanas y el abrigo Roc de Dourgne en el alto valle del Aude (GUILAINE et al. 1993) son algunos de los yacimientos más representativos. Se trata por lo general, de asentamientos que se caracterizan por una economía agro-pastoril ya plenamente desarrollada.

Por el contrario, no se han encontrado apenas vestigios arqueológicos fechados en este intervalo cronológico (5500-5300 cal ANE) ni en las sierras interiores ni en la zona Axial del Pirineo. En toda esa área, los indicios de actividad humana son aún muy débiles para esa primera etapa. La información procedente de turberas y lagos alpinos, tanto en la vertiente española como de la francesa, nos habla de un impacto antrópico mínimo, a veces difícilmente perceptible (CATALÁN et al. 2001, MIRAS et al. 2007, EJARQUE et al. 2010, PÈLACHS et al. 2011). Algunos autores consideran que en esta fase inicial de expansión del neolítico, las actividades humanas en alta montaña fueron demasiado discontinuas y efímeras para dejar una huella clara en los registros palinológicos (GALOP et al. 2013). Esta interpretación encaja bien con el registro arqueológico hallado en zonas de altura hasta la actualidad (GASSIOT et al. 2014, ORENGO et al. 2014). Se trata en definitiva de un momento en el cual las actividades humanas parecen concentrarse en las áreas más exteriores de la cordillera, alrededor de fondos de valle o en laderas de baja altitud. Los pisos montano y subalpino aún no serían explotados de forma sistemática, registrándose solo intervenciones marginales y discontinuas, además con notable variabilidad entre una y otra área del Pirineo (GALOP 2006).

Para encontrar ocupaciones a mayor altitud hay que esperar esperar hasta el intervalo del 5300-4600 cal ANE. Durante esta franja cronológica aparecen las primeras evidencias arqueológicas en las zonas más internas del Pirineo, es decir en la Depresiones Medias y en las Sierras Interiores. Estamos hablando de una variedad de contextos, desde grandes cuevas-rediles, hasta ocupaciones en pequeñas cavidades y abrigos y también de alguna estructura al aire libre. Algunos de los yacimientos más Ilamativos son la Cova Gran (380 msnm) (MORA et al. 2011) y Cova del Parco (PETIT 1996) (420 msnm) en los relieves más exteriores del Prepirineo de Lleida, Cova Colomera (670 msnm) (OMS et al. 2014) en la cadena del Montsec, el poblado de Sanavastre en el valle del Segre (1.080 msnm) (MERCADAL et al. 2009), la Balma Margineda en Andorra (900 msnm) (GUILAINE y MARTZLUFF 1995), la Espluga de la Puyascada en la Sierra Ferrera (1.315 msnm) (BALDELLOU 1987), la Cova de Els Trocs (1.530 msnm) (ROJO et al. 2014) y la recién excavada Cueva de Coro Trasito en el Prepirineo Aragonés (1.540 msnm) (CLEMENTE et al. 2014) y, a mayor altitud, la Cova del Sardo de Boí en la Alta Ribagorça (1.790 msnm). Junto con los datos paleobotánicos procedentes de lagos alpinos y turberas (MIRAS et al. 2007, EJARQUE et al. 2010, PÉREZ-OBIOL et al. 2012), estos descubrimientos nos permiten plantear una gradual presencia humana en las zonas de media y alta montaña desde finales del $\mathrm{VI}$ milenio y durante toda la primera mitad del $\mathrm{V}$ milenio cal ANE

Ahora bien, si la cronología de las primeras ocupaciones en las zonas de montaña del Pirineo queda bastante bien establecida, aún es argumento de debate la funcionalidad de estos asentamientos y las actividades económicas allí desarrolladas. ¿Se trata de ocupaciones pastoriles especializadas? ¿se practicaba la agricultura en estos lugares o en sus cercanías? ¿o acaso eran lugares de caza? De hecho hay muchas formas de llevar a cabo cada una de estas actividades; modalidades de gestión diferentes de los recursos que implican relaciones sociales diferentes a nivel intergrupal, así como diferentes modalidades de explotación y ocupación del territorio que implican patrones de movilidad y de asentamiento diferentes.

A pesar de que sean temas indudablemente muy complejos para resolver en arqueología, en los últimos años nos estamos acercando a una mejor comprensión de cómo los espacios montañosos pirenaicos fueron ocupados durante el Neolítico y de cómo estas áreas fueron integradas dentro de un sistema económico más amplio (MAZZUCCO 2014). En este trabajo vamos a llevar a cabo una reflexión sobre algunas de las prácticas de subsistencia de los primeros grupos agro-pastorales que ocuparon el Pirineo Central en el VI-V milenio cal ANE y, en particular, sobre las prácticas de caza y de cultivo, intentando profundizar en algunos de los datos disponible acerca de dichas actividades y de la organización económicas de esas poblaciones. 


\section{2.- CAZAR O NO CAZAR ¿AÚN ES ESTA LA CUESTIÓN?}

La presencia de grupos de cazadores-recolectores en las zonas de media y alta altura del Pirineo Central es un tema aún poco explorado y, en general, la información arqueológica al respecto es muy escasa. Lo que sabemos ciertamente es que a partir del Tardiglaciar y Holoceno inicial-momento en el cual una ocupación de las cotas altas hubiera sido posible tras la retirada de los últimos glaciares de valle- $(P E L A C H$ et al. 2012) se conocen numerosos asentamientos, con cronologías comprendida entre el 12.200-12.000 y el 6690-6570 cal ANE. Algunos de los más destacados son Balma Guilanyà, Font del Ros, Sota Palou, Cova del Parco, Forcas I, Forcas II y la Balma Margineda por la vertiente española (MARTÍNEZ-MORENO et al. 20062007), mientras por la vertiente francesa se conocen La Tourasse, Abri du Buholoup, Roc du Dourgne, Cova de Poeymaü, Grotte de Moulin, entre otros (MARTIN y VAQUER 1995) (Figura 1).

Sin embargo, se trata en todos los casos de contextos localizados en las sierras más exteriores del Pirineo y en altitudes inferiores a los 1000 metros. De hecho, a pesar de las numerosas campañas de prospección llevadas a cabo en los últimos diez años en diferentes sectores del Prepirineo y del Pirineo Central, hasta el día de hoy casi no se conocen yacimientos del Epipa- leolítico o Mesolítico en áreas de media y alta montaña. Sólo recientemente se ha dado a conocer el descubrimiento de algunos asentamientos de época Mesolítica en las franjas de mayor altitud del Pirineo Central. Un ejemplo es la ocupación Mesolítica encontrada en el valle de Perafita, en Andorra, a más de 2.000 metros de altitud; sin embargo, se trata de un yacimiento conocido únicamente a través de un sondeo y la caracterización de la ocupación tanto a nivel estratigráfico como a nivel tecnológico y económico es aún parcial (ORENGO et al. 2014). Igualmente, en el Pirineo catalán se conocen desde hace pocos años dos asentamientos con niveles fechados entre el 10700 y el 6600 cal ANE, respectivamente el Dolmen de la Font del Coms y el Abric del Estany de la Coveta I (GASSIOT et al. 2014); en ambos casos estamos ante ocupaciones que han dejado pocos restos materiales -entre los que no destacan armas de caza-y que, en todos los casos, no parecen responder a un modelo de frecuentación intensiva y reiterada de las alta cotas, sino más bien ocasional.

Sin profundizar en el asunto, esta breve digresión nos sirve para remarcar que, fundamentalmente, en base a los datos actuales no es posible evidenciar una explotación de las zonas de alta montaña del Pirineo Central para actividades de caza durante el final del Paleolítico, momento en el cual, al contrario, se generaliza la ocupación de los pisos subalpinos y alpinos por partes de grupos de cazadores en el resto de las

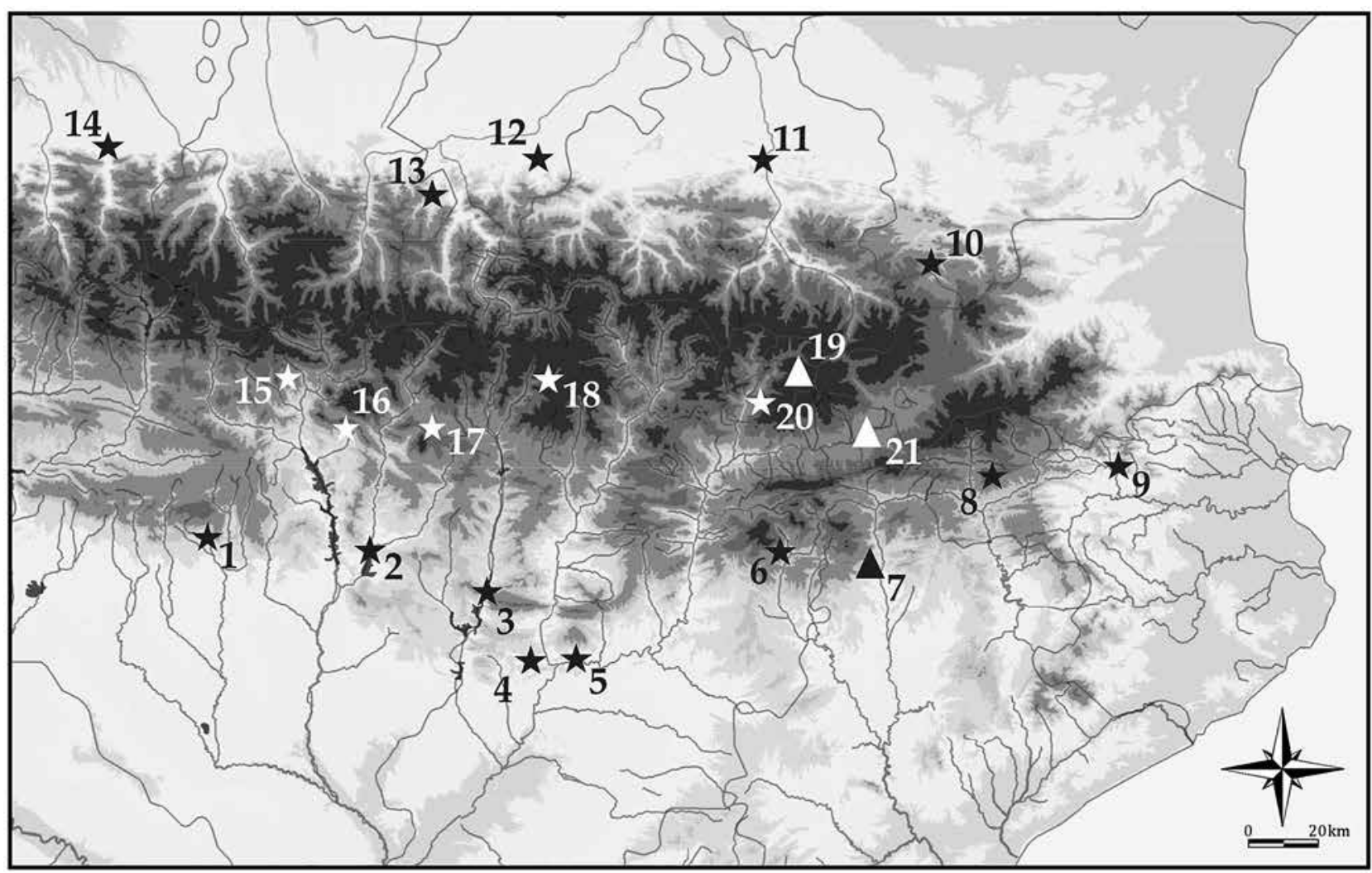

Fig. 1. Yacimientos citados en el texto. Las estrellas representan los asentamientos bajo roca, mientras que los triángulos los asentamientos al aire libre. 1) Cueva de Chaves; 2) Forcas II; 3) Cova Colomera; 4) Cova Gran; 5) Cova del Parco; 6) Balma Guilanyà; 7) Font del Ros; 8) Sota Palou; 9) Serrat del Pont; 10) Roc de Dourgne; 11) Abri de Buholoup; 12) Grotte de la Tourasse; 13) Abri du Moulin; 14) Grotte du Poeymaü; 15) Coro Trasito; 16) Espluga de la Puyascada; 17) Cova de Els Trocs; 18) Cova del Sardo; 19) Perafita; 20) Balma Margineda; 21) Sanavastre. 
montañas europeas en genreal y en los cercanos AIpes en particular (BROGLIO 1992, CROTTI y PIGNAT 1992; BINTZ y GRIGGO 2011). Únicamente la Balma Margineda, situada en el fondo del valle de la Valira, a 970 msnm, al sur de la Cordillera Pirenaica, presenta una ocupación reiterada durante el Epipaleolítico con numerosas evidencias de caza, sobretodo en relaccion a Capra pyreneaica (GUILAINE y MARTZLUFF 1995).

Contrariamente, en el Pirineo Central, las primeras evidencias de actividades cinegéticas en zonas de altitud se fechan en un momento avanzado del Neolítico antiguo y se relacionan con poblaciones ya caracterizadas por una economía agro-pastoral. ¿Podemos avanzar alguna hipótesis sobre que rol jugaba la caza en la economía de estos primeros pobladores? ¿Qué tipo de caza practicaban? ¿Cuáles eran sus principales objetivos?

Para llevar a cabo una primera evaluación de las evidencias disponible tomaremos en cuenta los datos faunísticos disponibles acerca de los ungulados salvajes cazados en los yacimientos del primer Neolítico pirenaico (Tabla 1.).

Ya a primera vista se observa que los herbívoros alpinos (es decir Capra pyreneaica y Rupicapra rupicapra) están escasamente representados y de forma discontinua entre los yacimientos considerados. El sitios donde presentan porcentajes más relevantes es Roc du Dourgne, llegando al 17\% de los restos de fauna salvaje en el nivel C6 y hasta el 34\% en el nivel C5. Con porcentajes menores, los herbívoros alpinos están representados también a la Cueva de Chaves, niveles 1b-1a, y en la Peña de Forcas II, nivel VI. Es interesante remarcar que en todo caso se trata de yacimientos periféricos respecto a la zona Axial de la cordillera y que probablemente pudieron aprovechar como áreas de caza los pisos subalpinos y alpinos de las mismas Sierras Margi-
nales/Exteriores, sin necesariamente adentrarse hacia el interior de la cordillera. En el resto de los asentamientos los animales mejor representados son las especies de bosque, en particular el ciervo (Cervus elaphus), que alcanza alrededor del $70-80 \%$ de los restos de ungulados salvajes, seguido por el jabalí (Sus scrofa), entre el $25-35 \%$ y el corzo (Capreolus capreolus), cerca del $13-14 \%$. De todas formas, hay que remarcar que, independientemente de la especie cazada, el número absoluto de restos de ungulados salvaje representan un conjunto bastante reducido en todos los contextos analizados, con un número mínimo de individuos que como máximo se establece alrededor de la decena. Los asentamientos con un número mayor de restos de animales salvajes son la Cueva de Chaves (nivel 1b1a), Peña de Forcas II (nivel V) y Roc du Dourgne (nivel C5-6), todos ellos localizados en las zonas marginales de la cordillera. En los yacimientos de las Sierras Interiores del Pirineo como la Cova de Els Trocs, la Espluga de la Puyascada y la Balma Margineda, se han recuperado conjuntos aún más reducidos. Además, en yacimientos de mayor altitud, como la Cova del Sardo (conjunto 8), no se han podido reconocer restos faunísticos de mamíferos salvajes y, en general, los conjuntos faunísticos han resultado numericamente pobres. Este escenario parece apuntar hacia un mayor consumo de fauna salvaje en los asentamientos localizados en las áreas exteriores del Pirineo, mientras que en las zonas alpina del Pirineo Axial y de las Sierras el consumo el parece disminuir de forma muy considerable.

Si confrontamos estos datos con los procedentes del análisis de la industria lítica (Tabla 2), observamos un panorama bastante similar, aunque se puede remarcar algún aspecto de interés. En casi todos los yacimientos considerados, los elementos microlíticos de retoque abrupto -principalmente compuestos por segmentos, trapecios y triángulos- representan una

\begin{tabular}{|c|c|c|c|c|c|c|c|c|c|c|c|c|c|c|c|}
\hline \multirow{2}{*}{ SITIOS } & \multicolumn{2}{|c|}{ Cervus elaphus } & \multicolumn{2}{|c|}{ Capreolus Capreolus } & \multicolumn{2}{|c|}{ Sus scrofa } & \multicolumn{2}{|c|}{ Bos Primigenius } & \multicolumn{2}{|c|}{ Equus ferus } & \multicolumn{2}{|c|}{ Capra Pyreneaica } & \multicolumn{2}{|c|}{ Rubicapra Rubicapra } & \multirow{2}{*}{ Tot } \\
\hline & $\varepsilon$ & $\%$ & $\varepsilon$ & $\%$ & $\Sigma$ & $\%$ & $\varepsilon$ & $\%$ & $\varepsilon$ & $\%$ & $\varepsilon$ & $\%$ & $\varepsilon$ & $\%$ & \\
\hline $\mathrm{CH} 1 \mathrm{~b}$ & 664 & 68,7 & 58 & 6,0 & 108 & 11,2 & 7 & 0,7 & 7 & 0,7 & 123 & 12,7 & 0 & 0,0 & 967 \\
\hline $\mathrm{CH} 1 \mathrm{a}$ & 393 & 73,7 & 29 & 5,4 & 46 & 8,6 & 5 & 0,9 & 5 & 0,9 & 55 & 10,3 & 0 & 0,0 & 533 \\
\hline TR 1 & 6 & 75,0 & 2 & 25,0 & $\mathrm{n} / \mathrm{a}$ & $n / a$ & 0 & 0,0 & 0 & 0,0 & 0 & 0,0 & 0 & 0,0 & 8 \\
\hline PUY E.II & 13 & 76,5 & 4 & 23,5 & 0 & 0,0 & 0 & 0,0 & 0 & 0,0 & 0 & 0,0 & 0 & 0,0 & 17 \\
\hline BM C.3 & 17 & 100,0 & 0 & 0,0 & $\mathrm{n} / \mathrm{a}$ & n/a & n/a & $\mathrm{n} / \mathrm{a}$ & 0 & 0,0 & 0 & 0,0 & 0 & 0,0 & 17 \\
\hline FO V & 222 & 85,4 & 17 & 6,5 & 15 & 5,8 & 2 & 0,8 & 1 & 0,4 & 0 & 0,0 & 3 & 1,2 & 260 \\
\hline FO VI & 56 & 72,7 & 10 & 13,0 & 2 & 2,6 & 1 & 1,3 & 0 & 0,0 & 2 & 2,6 & 6 & 7,8 & 77 \\
\hline DO 5 & 23 & 15,0 & 25 & 16,3 & 52 & 34,0 & 0 & 0,0 & 0 & 0,0 & 53 & 34,6 & 0 & 0,0 & 153 \\
\hline DO 6 & 22 & 15,9 & 27 & 19,6 & 65 & 47,1 & 0 & 0,0 & 0 & 0,0 & 24 & 17,4 & 0 & 0,0 & 138 \\
\hline SA 8 & $\mathrm{n} / \mathrm{a}$ & $\mathrm{n} / \mathrm{a}$ & $\mathrm{n} / \mathrm{a}$ & $\mathrm{n} / \mathrm{a}$ & $\mathrm{n} / \mathrm{a}$ & $\mathrm{n} / \mathrm{a}$ & n/a & $\mathrm{n} / \mathrm{a}$ & $\mathrm{n} / \mathrm{a}$ & $\mathrm{n} / \mathrm{a}$ & n/a & $\mathrm{n} / \mathrm{a}$ & $\mathrm{n} / \mathrm{a}$ & $\mathrm{n} / \mathrm{a}$ & $\mathrm{n} / \mathrm{a}$ \\
\hline
\end{tabular}

Tabla 1: Frecuencias de los diferentes ungulados salvajes en algunos de los yacimientos citados en el texto. n/a - indica que el dato no está disponible. To - indica la totalidad de restos calculada sobre los valores indicados en la misma tabla. $\mathrm{CH} 1 \mathrm{~b}$ y $\mathrm{CH} 1 \mathrm{a}-\mathrm{Cueva}$ de $\mathrm{CHaves}$, niveles $1 \mathrm{~b}$ y $1 \mathrm{a}$; TR 1 - Cova de Els Trocs, fase1. PUY E.II - Espluga de la Puyascada, nivel E.II; BM C.3 - Balma Margineda, nivel 3; FO V y FO VI - Peña de las Forcas II, niveles V y VI; DO 5 y 6 - Roc du Dourgne, niveles 5-6; SA 8 - Cova del Sardo, fase8 / Frecuencies of the faunal remains of the wild ungulated from some of the sites cited in the the text. $\mathrm{n} / \mathrm{a}$ - data it is not reported or available. Tot - totality of remains calculated on the values expressed in the table for each site/layer. $\mathrm{CH} 1 \mathrm{~b}$ y $\mathrm{CH}$ 1a - Cueva de CHaves, layers 1b y 1a; TR 1 - Cova de Els Trocs, phasel. PUY E.II - Espluga de la Puyascada, layer E.II; BM C.3 - Balma Margineda, layer 3; FO V y FO VI - Peña de las Forcas II, layers V y VI; DO 5 y 6 - Roc du Dourgne, layers 5-6; SA 8 - Cova del Sardo, phase8. 


\begin{tabular}{|c|c|c|c|}
\hline SITIOS & $n^{\circ}$ microlítos & \% retocados & $\%$ Tot \\
\hline CH 1b & 44 & 9,8 & 2,8 \\
\hline CH 1a & 9 & 3,2 & 1,1 \\
\hline TR 1 & 7 & 33,3 & 6,3 \\
\hline PUY E.II & 0 & 0 & 0 \\
\hline BM C.3 & 18 & 9,2 & 1,6 \\
\hline FO V & 41 & 44,5 & 5,3 \\
\hline FO VI & 11 & 35,5 & N/A \\
\hline DO 5 & 34 & 29,8 & 4,6 \\
\hline DO 6 & 22 & 26,2 & 3,8 \\
\hline SA 8 & 2 & 40,0 & 3,4 \\
\hline
\end{tabular}

Tabla 2: Frecuencias de los microlítos recuperados en algunos de los yacimientos citados en el texto. n/a - indica que el dato no está disponible. \% Tot - indica la porcentual de microlítos calculada sobre el número total de restos líticos por cada nivel. $\mathrm{CH} 1 \mathrm{~b}$ y $\mathrm{CH} 1 \mathrm{a}-$ Cueva de CHaves, niveles 1b y 1a; TR 1 - Cova de Els Trocs, fase1. PUY E.II - Espluga de la Puyascada, nivel E.II; BM C.3 - Balma Margineda, nivel 3; FO V y FO VI - Peña de las Forcas II, niveles V y VI; DO 5 y 6 - Roc du Dourgne, niveles 5-6; SA 8 - Cova del Sardo, fase8 / Frecuencies of the backed tolos recovered from some of the sites cited in the the text. n/a - data it is not reported or available. \% Tot - totality of remains calculated on the values expressed in the table for each site/layer. $\mathrm{CH} 1 \mathrm{~b}$ y $\mathrm{CH} 1 \mathrm{a}-$ Cueva de CHaves, layers $1 \mathrm{~b}$ y 1a; TR 1 - Cova de Els Trocs, phasel. PUY E.II - Espluga de la Puyascada, layer E.Il; BM C.3 - Balma Margineda, layer 3; FO V y FO VI - Peña de las Forcas II, layers V y VI; DO 5 y 6 - Roc du Dourgne, layers 5-6; SA 8 - Cova del Sardo, phase8.

porción bastante relevante del utillaje retocado. Chaves es uno de los sitios donde estos tipos tienen menor peso, respectivamente el 9,8\% y el 3,2\% de la industria en los niveles 1b y 1a. Porcentajes similares se evidencian también en la Balma Margineda $(9,2 \%)$ y en Els Trocs-fase II (6,6\%). En los demás asentamientos, los geométricos tienen una importancia mucho más marcada respecto a la estructura tipológica, con una porcentaje promedio del 34\%, hasta un máximo del $44 \%$ en Forcas II-nivel 5. Sin embargo, si limitamos nuestro análisis únicamente a los elementos retocados, corremos el riesgo de sacar una lectura demasiado parcial: de hecho, si nos fijamos en la totalidad de la industria lítica, yacimiento por yacimiento, podemos ver que en todos los contextos analizados la producción de instrumentos de caza (pudiéndose generalizar esta función a la mayoría de los elementos microlíticos geométricos y a las laminillas de dorso abatido), representa una porción más bien reducida, con un porcentaje promedio alrededor del $4 \%$ de los restos. Este escenario parece sugerir que, a pesar de que sean una de las categorías de útiles formales más importantes, con respecto a la globalidad de la producción lítica tallada, las armaduras juegan un papel secundario o, igualmente, no tan relevante.

En definitiva, el dato que extraemos al considerar tanto los restos faunístico cuanto los restos líticos es que las actividades vinculadas a la caza -es decir la producción y mantenimiento de elementos de proyectil y el consumo de animales salvajes- tuvieron un papel más bien marginal dentro de la economía de las ocupaciones del primer Neolítico pirenaico. Además, hay que remarcar que contrariamente a lo esperado no se observa un incremento de las actividades cinegéticas en los contextos de mayor altitud; de hecho, las ocupaciones con indicios de caza más claros son el nivel $\mathrm{V}$ de la Peña de las Forcas II y en los niveles 5 y 6 de Roc du Dourgne, todos ellos localizados en las franjas más externas del Pirineo, con altitudes inferiores a los 1.000 metros. Entre éstos, el sitio que presenta una funcionalidad más claramente enfocada hacia las actividades cinegéticas es seguramente Forcas II-nivel V. Sin embargo se trata de un contexto que no es posible considerar 'Neolítico' y que, consecuentemente, tal vez se tendría que excluir del actual análisis. De hecho, sus excavadores sugieren una ocupación del abrigo por parte de grupos de cazadores-recolectores, con una cultura material fundamentalmente mesolítica, en las que se integrarían raros elementos neolíticos, principalmente representados por fragmentos de cerámica y algunas técnicas de retoque (UTRILLA y MAZO 2014). En esta línea, los dos autores hablan de una ocupación enfocada hacia la caza y al descuartizado y consumo de las presas cazadas, principalmente ciervos. Actualmente es este el único contexto del periodo que se puede considerar especializado en actividades cinegéticas.

Finalmente, por lo que concierne la Cova de Els Troc-fase I y la Cova del Sardo-nivel 7 a pesar de que en ambos casos los geométricos representen porcentajes relevantes del material lítico tallado. Aunque estamos hablando de conjuntos numéricamente muy reducidos, alrededor de un centenar de piezas líticas incluyendo tanto desechos como útiles y en los que se contabilizan menos de una decena de elementos de proyectil. De lo cual se deduce que también en estos casos parecería más apropiado hablar de una explotación cinegética no sistemática o intensiva, sino más bien ocasional. Además, la escasez o ausencia de fauna salvaje en ambos yacimientos parece confirmar este hecho.

\section{3.- AGRICULTURA EN ZONAS DE ALTA MON- TAÑA DURANTE EL NEOLÍTICO ANTIGUO: UN ESTADO DE LA CUESTIÓN}

El estudio de las prácticas agrícolas en zonas de media y alta montaña durante el Neolítico es un tema de debate muy actual, no solo porqué nos ayuda a comprender las formas de explotación del territorio de montaña a lo largo del tiempo, sino que también nos permite reconstruir las formas de vida de estos primeros pobladores y su organización económica y social. Ya hemos mencionado que las primeras evidencias de la llegada de poblaciones agro-pastorales al área pirenaica nos indican la implantación de un modelo económico mixto, que incluía -ya desde las primeras etapas- una producción agrícola y una producción ganadera bien establecidas (MAZZUCCO et al. 2015). Sin embargo, este modelo no se puede reconocer en todos los asentamientos del área, sino solo en algunas ocupaciones de mayor entidad y extensión, poblados al aire 
libre o grandes cuevas. Por lo que concierne a las otras ocupaciones y en particular a los asentamientos localizados a mayor altitud ¿qué informaciones tenemos?, ¿se trata de ocupaciones secundarias, especializadas, o sencillamente de ocupaciones relacionadas a pequeños grupos que repetían el mismo modelo económico, pero a menor escala, incluyendo tanto una producción agrícola como ganadera?

Durante los últimos diez años numerosos estudios de carácter paleoambiental han logrado demostrar que el desarrollo de áreas de cultivo en zonas de alta montaña fue una práctica común también en épocas prehistóricas (PÉLACHS et al. 2007). En el Pirineo Central, a través de sondeos de paleosuelos y estudios de columnas sedimentarias de lagos y turberas, se ha evidenciado la presencia de relevantes porcentajes de polen de cereal y de otros macro- y micro-restos vegetales domésticos a altitudes superiores a los 2.000 msnm, sugiriendo que la agricultura fue practicada en las altas cotas y en los fondos de valle alpinos en época Medieval, Romana e incluso en épocas protohistóricas (MIRAS et al. 2007, BAL et al. 2011). Este escenario bien encaja con la información recolectada gracias a las extensivas campañas de prospecciones realizadas en diferentes áreas del Pirineo. Sin entrar al detalle de las evidencias los vestigios encontrados nos hablan de un intenso poblamiento de las altas cotas a partir como mínimo del II-III siglo cal NE (GARCIA-CASAS 2013, ORENGO et al. 2013), hasta llegar a un momento de máxima presión demográfica durante la Edad Media, siglos IX-XIII cal NE (RENDU 2003, GARCIA-CASAS 2013).

Sin embargo, para el Neolítico el panorama arqueológico y paleoambiental parece bastante diferente. Los estudios polínicos nos hablan de una presión antrópica mucho más débil, aún intermitente, discontinua en el tiempo y dispersa en el espacio (GALOP 2006). De hecho, en base a los datos polínicos obtenidos en diferentes áreas de estudio, después de las primeras señales de impacto sobre el medio natural, alrededor del 55005300 cal ANE, se detectaría una fase de recuperación del bosque (MIRAS et al. 2007, EJARQUE et al. 2012, PÈREZ-OBIOL et al. 2012). La existencia de ciertas discontinuidades en la ocupación de los espacios de montaña, incluso de un mismo valle, está bien atestiguada por la sucesión de las fases de ocupación del yacimiento arqueológico de la Cova del Sardo (GASSIOT et al. 2012). Además de una intermitencia temporal, hay que destacar una baja concentración de evidencias arqueológicas en el espacio, sobre todo para la primera etapa del Neolítico, entre la segunda mitad del VI y la primera mitad del V milenio cal ANE. Sin duda, la falta de prospecciones sistemáticas en la gran mayoría del Pirineo central, representa un límite a la reconstrucción de los modos de ocupación del espacio por parte de las primeras comunidades agro-pastorales. Igualmente, hay que destacar que también en áreas más intensamente prospectadas, como el Parque Nacional de Aigüestortes i Estany de Sant Maurici o los valles de
Madriu-Perafita-Claror en Andorra, las evidencias arqueológicas que remontan hasta este periodo son muy escasas, sugiriendo una baja presión demográfica, almenos en las áreas de alta montaña.

No obstante, hay algunas evidencias que se vinculan a las prácticas agrícolas, tanto a nivel paleoambiental como arqueológico, en los yacimientos del primer Neolítico pirenaico (5500-4600 cal ANE). De hecho, en casi todos los contextos arqueológicos del periodo se han encontrado restos carbonizados de cereales: en la Balma Margineda se han recuperado restos de trigo (Triticum sp.) y cebada (Hordeum vulgare) (GUILAINE \& MARTZLUFF 1995); en la Cova de Els Trocs se ha recuperado una gran variedad de restos de plantas domésticas, entre los cuales destaca el trigo desnudo (Triticum aestivum/durum), la cebada (Hordeum vulgare) y las leguminosas, como lentejas (Lens culinaris) y guisantes (Pisum sativum) (LANCELOTTI et al. 2013). En la Cova de Sardo-fase8 se han documentado restos de cebada desnuda (Hordeum vulgare L. var. nudum Hook. f) (GASSIOT et al. 2014). Se trata en todos los casos de conjuntos muy reducidos que más bien nos indican un consumo de cereales in situ y no necesariamente su procesamiento o su cultivo. De hecho, en el único yacimiento donde se ha llevado a cabo un estudio de detalle sobre los microrestos vegetales, la Cova de Els Trocs, se ha demostrado que el procesamiento de los granos no tuvo lugar in situ y que los restos recuperados se han de interpretar como desechos del consumo de provisiones traídas desde otro lugares (LANCELOTTI et al. 2013). La misma interpretación se ha dado también a los restos de plantas cultivadas encontrados en la Balma Margineda, donde además del consumo, se ha atestiguado también la presencia de probables fosas de almacenaje (GUILAINE Y MARTZLUFF 1995).

Además de los marcadores arqueobotánicos se han encontrado también algunos útiles en sílex asociados con la recolección de los cereales. Se han recuperado elementos de hoz en la Espluga de la Puyascada (MAZZUCCO et al. 2013a), en la Cova del Sardo (MAZZUCCO et al. 20 14a) y en la Cova de Els Trocs (ROJO et al. 2013). También en este caso se trata piezas aisladas y no de una producción y utilización sistemática de estos tipos de útiles. Además en algunos de estos asentamientos, como la Espluga de la Puyascada y la Cova del Sardo, a través de estudios funcionales de detalle, ha sido posible demostrar que la utilización de estos soportes como elementos de hoces ha sido previa a su transporte y deposición en el sitio (MAZZUCCO 2014). Los útiles de siega eran probablemente objetos que se solían mover y desplazar de un yacimiento al otro y, a la ocasión, se reutilizaban para otras actividades o para la recolección de otras plantas; su presencia por tanto, sobre todo si es limitada a pocos elementos, no tiene porqué implicar que las actividades de recolección de cereales se llevaran a cabo cerca del yacimiento.

En definitiva, durante las primeras fases del Neolítico no se puede hablar de prácticas de cultivo en zonas 
de media y alta montaña o por lo menos las evidencias hasta ahora no apoyan esta hipótesis. Sin embargo los datos apenas expuestos demuestran que estos grupos practicaban agricultura y consumían sus productos $\mathrm{y}$, en consecuencia, su modelo económico no parece destacarse del modelo previamente expuesto, es decir de un economía agro-pastoral mixta. El descubrimiento de nuevos asentamientos, especialmente de ocupaciones al aire libre, podría cambiar este escenario, pero hasta el momento no hay información al respecto. De hecho, los primeros poblados Neolíticos en zonas de montaña, alrededor de 1.300 msnm, se fecharían en el Neolítico medio, como se ha documentado en el Camp del Colomer, en Juberri, Andorra (ANTOLíN et al. 2015). Futuras investigaciones nos ayudarán a explicar estas cuestiones.

\section{4.- A MODO DE CONCLUSIÓN}

La ocupación de las cotas media y altas del Pirineo Central es un proceso relativamente lento y gradual y a pesar que los primeros indicios remonten al final del $\checkmark$ milenio cal ANE, una antropización más intensiva del medio montañoso no será evidente antes del III milenio cal ANE. En las primeras etapas, aproximadamente entre el 5500 y el 4600 cal ANE, observamos la presencia dispersa de grupos humanos tanto en los pisos montanos y subalpinos de la cordillera, con altitudes desde los 400 hasta los $1.800 \mathrm{msnm}$. Se trata principalmente de ocupaciones en cuevas o abrigos de diferente tamaños y extensión. Por el momento no se conocen asentamientos al aire libre para ese periodo. De todas formas, en este sentido hay que remarcar que la representación de los sitios está fuertemente influenciada por las técnicas de prospección empleadas y la escasez de grande obras de infraestructuras o edificación en el área de estudio.

Respecto a los asentamientos localizados en las franjas marginales de la cordillera (ej. Cueva de Chaves, Font del Ros, etc.), los yacimientos que se hallan en los pisos montanos y subalpinos (Balma Margineda, Cova del Sardo, Espluga de la Puyascada, Cova de Els Trocs, etc.), evidencian un carácter mayormente especializado. Su vocación económica aparece vinculada principalmente al desarrollo de actividades ganaderas de diferente naturaleza, desde la creación de nuevas áreas de pastos, hasta la estabulación de los animales, su matanza y, tal vez, el procesamiento y transformación de sus productos. Hemos visto que tanto la caza como la agricultura están representadas entre las actividades de subsistencias, pero sin practicarse de forma intensiva en estos contextos. Sin embargo, no se trata de grupos humanos separados, que siguen un modelo de subsistencia diferente respecto a los grupos humanos localizados a menores altitudes; es decir no se puede plantear una 'oposición' entre grupos pastoriles y comunidades de agricultores. Al contrario, todas estas ocupaciones son expresiones del mismo sistema económico -una economía mixta agropecuaria- y de su desarrollo en el espacio físico. De hecho, el transporte de provisiones alimenticias y de útiles y artefactos líticos de un yacimiento hacia otro (o de un área a otra) antes mencionado, nos sugiere la existencia de una movilidad inter-sitios y de una organización espacial de las actividades productivas y a la vez de sus productos. Asimismo, los estudios realizados sobre las materias primas líticas de varios asentamientos de la vertiente meridional del Pirineo Central (MAZZUCCO et al. 2013b y 2014b), en particular de las rocas silíceas, nos ofrece un cuadro fundamentalmente idéntico: los grupos neolíticos se movieron y explotaron recursos distribuidos sobre un territorio relativamente amplio, que incluye zonas de altura pero también áreas localizadas a menores altitudes, tales como las Sierras Marginales/Exteriores y la depresión del Ebro, o al menos de su sector más septentrional. Hasta el momento no podemos afirmar si parte de las comunidades que habitaban las franjas externas del Pirineo se desplazaban de forma estacional hacia las zona Axial de la cordillera o si al contrario existían grupos que habitaban todo el año en los valles pirenaicos más internos, desplazándose entre zonas de fondo de valle y alta montaña (o si ambos modelos coexistieron). Está claro de que se trata de un punto crucial para poder profundizar nuestro conocimiento sobre la organización de dichas sociedades, sobre los modelos ganaderos vigentes y las formas de gestión y ocupación del territorio. Hace falta mucha más información, es decir más trabajos de excavación, más prospecciones y más análisis de materiales arqueológicos. De todas formas, los avances realizados durante las últimas décadas por los diferentes equipos de investigación, representan un punto de partida para plantear nuevas hipótesis y líneas de trabajo.

\section{AGRADECIMIENTOS}

Este trabajo se ha realizado en el marco de la tesis doctoral de uno de los autores, financiada por el programa 'JAE-pre' del CSIC y desarrollada en la Institución Milà y Fontanals de Barcelona y en la Universidad Autonoma de Barcelona. Este trabajo se inserta en los proyectos: Análisis ecológico de la culturización del paisaje de alta montaña desde el Neolítico: los Parques Nacionales de montaña como modelo (CUL-PA) (2013-2016), financiado por el Ministerio de Agricultura, Alimentación y Medio Ambiente y Modelizacion de los espacios prehistoricos de montaña. Un sig del patrimonio arqueologico y los territorios pastoriles (2016-2018) financiado por el Ministerio de Economía y Competitividad.

\section{BIBLIOGRAFÍA}

ALCALDE, G., MOLIST, M., SAÑA, M.

2005 Les Ocupacions neolítiques de la Bauma del Serrat del Pont (la Garrotxa). Tribuna d'Arqueologia 2001-2002, 27-39. 
ANTOLÍN, F., JACOMET, S., BUXO, R.

2015 The hard knock life. Archaeobotanical data on farming practices during the Neolithic $(5400 \mathrm{e} 2300$ cal BC) in the NE of the Iberian Peninsula. Journal of Archaeological Science 61, 90-104.

\section{BALDELLOU, V.}

1987 Avance al estudio de la Espluga de la Puyascada. Bolskan 4, 3-42.

\section{BALDELLOU, P., UTRILLA, P. \& GARCÍA-GAZÓLAZ, J.}

2012 Variscita de Can Tintorer en el Neolítico antiguo del Valle medio del Ebro, in BORRELL, M., BORRELL, F., $\mathrm{BOSCH}$, A., CLOP, X., MOLIST, M. (Eds.). Actes del Congrés Internacional: Xarxes al Neolític. 307-214. Rubricatum 5 .

BAL, M., PELACHS, A., PÉREZ-OBIOL, R., JULIA, R. \& CUNILL, R.

2011 Fire history and human activities during the last $3300 \mathrm{cal}$ yr BP in Spain's Central Pyrenees: The case of the Estany de Burg. Palaeogeography, Palaeoclimatology, Palaeoecology 300(1), 179-190.

BINTZ, P. et GRIGGO, C.

2011 Climats et premiers peuplements des Alpes du Nord françaises: des derniers chasseurs aux premiers paysans (15000 à 5000 ans av. JC.). Revue de primatology 3. Available in: http:/primatologie.revues.org/789.

BROGLIO, A.

1992 Mountain sites in the context of the north-eastltalian Upper Palaeolithic and Mesolithic. Preistoria Alpina 28, 293-310.

CATALÁN, J., PÉREZ-OBIOL, R. et PLA, S.

2001 Canvis climàtics a Aigüestortes durant els darrers 15.000 anys", en V Jornades sobre Recerca al Parc Nacional d'Aigüestortes i Estany de Sant Maurici (2000). 45-51. Departament de Medi Ambient. Lleida.

CLEMENTE, I., GASSIOT, E., REY, J., MAZZUCCO, N. y OBEA, L.

2014 "Cort o Transito"- Coro Trasito - O Corral de Tránsito: Una cueva pastoral del Neolítico antiguo en el corazón de Sobrarbe, in CLEMENTE, I., GASSIOT, E. \& REY, J. (Eds.). Sobrarbe antes de Sobrarbe: pinceladas de historia de los Pirineos, Centro de Estudios de Sobrarbe (CES) e Instituto de Estudios Altoaragoneses (IEA). 1132. Cometa S.A. Zaragoza.

CROTTI, P. et PIGNAT, G.

1992 L'utilisation des etages montagnards durant le Mesolithique dans les Alpes suisses. Preistoria Alpina 28, 275-284.

EJARQUE, A., MIRAS, Y., RIERA, S., PALET, J.M. \& ORENGO, H.A.

2010 Testing micro-regional variability in the Holocene shaping of high mountain cultural landscapes: a palaeoenvironmental case-study in the eastern Pyrenees. Journal of Archaeological Science 37(7), 1468-1479.

\section{GALOP, D.}

2006 La conquête de la montagne pyrénéenne au Néolithique, en GUILAINE, J. (Coord.). Populations Néolithiques et enivrements. 279-295. Ed. Errance. Paris.
GALOP, D., RIUS, D., CUGNY, C. et MAZIER, F.

2013 A History of Long-Term Human-Environment Interactions in the French Pyrenees Inferred from the Pollen Data, in LOZNY, L. R. (Ed.). Continuity and Change in Cultural Adaptation to Mountain Environments: From Prehistory to Contemporary Threats. 19-30. Springer. London. Studies in Human Ecology and Adaptation 7

GASSIOT, E., RODRÍGUEZ-ANTÓN, D., BURJACHS, F., ANTOLIN, F. y BALLESTEROS, A.

2012 Poblamiento, explotación y entorno natural de los estadios alpinos y subalpinos del Pirineo central durante la primera mitad del Holoceno. Cuaternario y Geomorfología 26(3-4), 29-45.

GASSIOT, E., RODRÍGUEZ ANTÓN, D., PÈLACHS, A., PÉREZ OBIOL, R., JULIÄ, R., BAL, M., C. y MAZZUCCO, N

2014 La alta montaña durante la Prehistoria: 10 años de investigación en el Pirineo catalán occidental. Trabajos de Prehistoria 71(2), 262-282.

GASSIOT, E., MAZZUCCO, N., OBEA, L., TARIFA, N., ANTOLÍN, F., CLOP, X., NAVARRETE, V., SAÑA, M. y RODRÍGUEZ, D.

2015 La Cova del Sardo de Boí i l'explotació de l'alta muntanya als Pirineus occidentals en època neolítica. Tribuna d'arqueologia 2013-2014, 199-218. Disponible en: http://hdl.handle.net/10687/122543.

GARCIA-CASAS, D.

2013 Aproximación al poblamiento de las zonas de alta montaña pirenaicas desde la arqueología y la etnografía. Sagvntvm 45, 221-239.

GUILAINE, J., BARBAZA, M., GASCO, J., GEDDES, D., COULAROU, J., VAQUER, J., ANDRE, J., JALUT, G. et VERNET, J.L.

1993 Dourgne: derniers chasseurs-collecteurs et premiers éleveurs de la Haute-Vallée de l'Aude. Centre d'anthropologie des sociétés Rurales, Archeologie en Terre d'Aude. Carcassonne.

GUILAINE, J., MARTZLUFF, M., GEDDES, D., COULAROU, J. et LE GALL, O.

1985 Postglacial Enviroments, Settlement and Subsistence in the Pyrenees: the Balma Margineda, Andorra, en BONSAL, C. (Ed.). Mesolithic in Europe. 561-571. Edinbourgh.

GUILAINE, J., y MARTZLUFF, M. (Eds.)

1995 Les excavacions a la Balma de la Margineda v. III. Govern d'Andorra. Andorra.

MARTÍN, A. et VAQUER, J.

1995 El poblament dels Pirineus a l'Holocé, del Mesolític al'Edat del Bronze, in BERTRAN PETIT, J. \& VIVES, E. (Eds.). Muntanyes i població. El passat dels Pirineus des d'una perspectiva multidisciplinària. 35-73. Ministeri de Relacions Exteriors, Centre de Trobada de les Cultures Pirinenques. Andorra.

LANCELOTTI, C., BALBO, A.L., MADELLA, M., IRIARTE, E., ROJO-GUERRA, M., ROYO, J.I., TEJEDOR, C., GARRIDO, R., GARCÍA, I., ARCUSA, H., PÉREZ-JORDÂ, G. \& PENNA-CHOCARRO, L.

2014 The missing crop: investigating the use of grasses at Els Trocs, a Neolithic cave site in the Pyrenees (1564 m asl). Journal of Archaeological Science 42, 456-466. 
MARTÍNEZ-MORENO, J., MORA, R. y CASANOVA, J.

2006/07 El contexto cronométrico y tecno-tipológico durante el Tardiglaciar y Postglaciar de la vertiente sur de los Pirineos orientales". Revista d'Arqueologia de Ponent $16-17,7-44$

MAZZUCCO, N.

2014 The Human Occupation of the Southern Central Pyrenees between the Sixth凶Third Millennia cal BC: a traceological analysis of flaked stone assemblages. PhD Dissertation. Universitat Autonoma de BarceIona. Barcelona. Available in: www.tdx.cat/handle/10803/287893

MAZZUCCO, N., CLEMENTE-CONTE, I., BALDELLOU, V. \& GASSIOT, E.

2013a The management of lithic resources during the $V$ millennium cal BC at the Espluga de la Puyascada (La Fueva, Huesca). Preistoria Alpina 47, 17-30.

MAZZUCCO, N., GASSIOT, E., ORTEGA, D., CLEMENTE, I. RODRÍGUEZ ANTÓN, D.

2013b Lithic procurement at the Cova del Sardo during Neolithic: preliminary data on mobility strategies. Archeologia Postmedievale 17, 87-96.

MAZZUCCO, N., ORTEGA, D., CLEMENTE, I., GASSIOT, E., BALDELLOU, B. \& ROJO GUERRA, M.

2014b Pautas de movilidad en el Pirineo central durante el Neolítico antiguo: una aproximación a partir de los recursos líticos, in CLEMENTE, I., GASSIOT, E. \& REY, J. (Eds.). Sobrarbe antes de Sobrarbe: pinceladas de historia de los Pirineos. 107-126. Centro de Estudios de Sobrarbe (CES) e Instituto de Estudios Altoaragoneses (IEA). Zaragoza.

\section{MAZZUCCO, N., GASSIOT, E., CLEMENTE, I.}

2014a The Human Occupation of the Mountain Environment the contribution of microwear analysis to the study of the Cova del Sardo site (Spanish Pyrenees), in MARREIROS, J., BICHO, N. \& GIBAJA, F.J. (Eds.). Usewear 2012: Proceedings of the International Conference on Use-wear Analysis. 329-340. Cambridge Scholar Publishing. Cambridge.

\section{MAZZUCCO, N., CLEMENTE, I., GASSIOT, E. \& GIBAJA, J.F.}

2015 Insights into the economic organization of the first agro-pastoral communities of the NE of the Iberian Peninsula: a traceological analysis of the Cueva de Chaves flaked stone assemblage. Journal of Archaeological Science. Reports 2, 353-366.

MERCADAL, O., ALIAGA I RODRIGO, S., ESTEVE, X., MANGADO, X., NADAL I., LORENZO, J., CUBERO CORPAS, C. \& ALVAREZ ARZA, $R$

2009 L'hàbitat del neolític antic de les mines de Sanavastre (Sanavastre, Das, la Cerdanya), in MERCADAL, O. (Ed.). XIV Col-loqui Internacional d'Arqueologia de Puigcerdà, 10-12 de novembre de 2006. Els Pirineus I les àrees circumdants durant el tardiglacial: mutacions i filiacions tecnoculturals, evolució paleoambiental (16000-10000 BP). 637-684. Institut d'Estudis Ceretans. Puigcerdà.
MIRAS, Y., EJARQUE, A., RIERA, S., PALET, J.M., ORENGO, $H$. et EUBA, I.

2007 Dynamique holocène de la végétation et occupation des Pyrénées andorranes depuis le Néolithique ancien, d'après l'analyse pollinique de la tourbière de Bosc dels Estanyons (2180m, Vall del Madriu, Andorre). Comptes Rendus Palevol 6(4), 291-300.

MORA, R., TERRADAS, X., PARPAL, A., PLANA, C., MARTÍNEZ, J., FÍGOLS, F. \& ROCA, G.

1991 Les ocupacions mesolítica i neolítica de la Font del Ros (Berga, Berguedà). Tribuna d'Arqueologia 1989-1990, 51-62.

MORA, R., BENITO CALVO, A., MARTÍNEZ MORENO, J., MARCEN, P.G. \& DE LA TORRE, I.

2011 Chrono stratigraphy of the Upper Pleistocene and Holocene archaeological sequence in Cova Gran (south eastern Pre Pyrenees, Iberian Peninsula). Journal of Quaternary Science 26(6), 635-644.

OMS, X. LÓPEZ-GARCÍA, J.M., MANGADO, X., MARTÍN, P., MENDIELA, S., MORALES, J.I., PARDO, M., RODRÍGUEZ, A., RODRÍGUEZ-CINTAS, A. \& YUBERO, M.

2013 Hàbitat en cova i espai pels ramats ca. 6200-6000 BP dades preliminars de la Cova Colomera (Prepirineu de Lleida) durant el neolític antic. Sagvntvm 45, 25-38.

ORENGO, H.A., PALET, J.M., EJARQUE, A., MIRAS, Y. \& RIERA, S.

2013 Pitch production during the Roman period: an intensive mountain industry for a globalised economy? Antiquity 87(337), 802-814

2014 Shifting occupation dynamics in the Madriu-Perafita-Claror valleys. Quaternary International 353, 140-152.

PÈLACHS, A., SORIANO, J.M., NADAL, J. \& ESTEBAN, A.

2007 Holocene environmental history and human impact in the Pyrenees. Contributions to Science 3(3), 423-431.

PÈLACHS, A., JULIÀ, R., PÉREZ-OBIOL, R., SORIANO, J.M., BAL, M.C., CUNILL, R. y CATALAN, J.

2011 Potential influence of Bond events on mid-Holocene climate and vegetation in southern Pyrenees as assessed from Burg lake LOI and pollen records. The Holocene 21(1), 95-104.

PÈLACHS, A., RODRÍGUEZ, J.M., PÉREZ-OBIOL, R., JULIÀ, R., BURJACHS, F., EXPÓSITO, I., CUNILL, R., SORIANO, J.M. \& YLL, R.

2012 Dinámica del clima y del paisaje vegetal del pirineo de Lleida durante la transicion TardiglaciarखHoloceno. Cuaternario y Geomorfología 26(3-4), 79-96.

PETIT, M. (Ed.)

1996 El procés de neolitització de la vall del Segre. La cova del Parco (Alòs de Balaguer, La Noguera): estudi de les ocupacions humanes del Vés al Iln mil.lenni a.C. Monografies del SERP, 1. Universitat de Barcelona. Barcelona.

PÉREZ-OBIOL, R., BAL, M-Cl., PÈLACHS, A., CUNILL, R., SORIANO, J.M.

2012 Vegetation dynamics and anthropogenically forced changes in the Estanilles peat bog (southern Pyrenees) during the last seven millennia. Vegetation History and Archaeobotany 21, 385-396. 
RENDU, C.

2003 La montagne d'Enveig: une estive pyreneen dans la longue durée. Ed. Trabucaire. Perpinyà.

ROJO, M.A., PEÑA-CHOCARRO, L., ROYO, J.I., TEJEDOR-RODRÍGUEZ, C., GARCÍA-MARTÍNEZ DE LAGRÁN, I., ARCUSA-MAGALLÓN, H., GARRIDO-PENA, R., MORENO-GARCÍA, M., MAZZUCCO, N., GIBAJA, J.F., ORTEGA, D., KROMER, B. y ALT, K.W.

2013 Pastores trashumantes del Neolítico Antiguo en un entorno de alta montaña: secuencia crono-cultural de la Cova de Els Trocs (San Feliú de Veri, Huesca). BSAA arqueología LXXIX, 9-55.

UTRILLA, P., y MAZO, C

2014 La Peña de las Forcas (Graus, Huesca). Un asentamiento estratégico en la confluencia del Ésera y el Isábena. Monografías Arqueológicas, Prehistoria 46. Universidad de Zaragoza. Zaragoza. 